\title{
Putting the Squeeze on Lead lodide Perovskites: Pressure-Induced Effects To Tune Their Structural and Optoelectronic Behavior
}

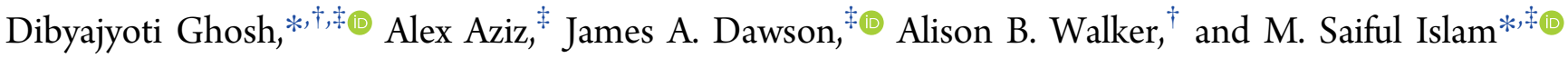 \\ ${ }^{\dagger}$ Department of Physics and ${ }^{\ddagger}$ Department of Chemistry, University of Bath, Bath BA2 7AY, U.K.
}

Supporting Information

\begin{abstract}
Lattice compression through hydrostatic pressure has emerged as an effective means of tuning the structural and optoelectronic properties of hybrid halide perovskites. In addition to external pressure, the local strain present in solution-processed thin films also causes significant heterogeneity in their photophysical properties. However, an atomistic understanding of structural changes of hybrid perovskites under pressure and their effects on the electronic landscape is required. Here, we use high level $a b$ initio simulation techniques to explore the effect of lattice compression on the formamidinium (FA) lead iodide compound, $\mathrm{FA}_{1-x} \mathrm{Cs}_{x} \mathrm{PbI}_{3}(x=0,0.25)$. We show that, in response to applied pressure, the $\mathrm{Pb}-\mathrm{I}$ bonds shorten, the $\mathrm{PbI}_{6}$ octahedra tilt anisotropically, and the rotational dynamics of the $\mathrm{FA}^{+}$ molecular cation are partially suppressed. Because of these structural distortions, the compressed perovskites exhibit band gaps that are narrower (red-shifted) and indirect with spin-split band edges. Furthermore, the shallow defect levels of intrinsic iodide defects transform to deep-level states with lattice compression. This work highlights the use of hydrostatic pressure as a powerful tool for systematically modifying the photovoltaic performance of halide perovskites.
\end{abstract}

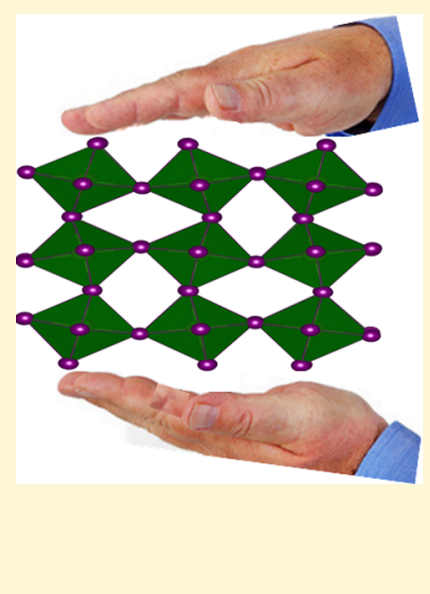

\section{INTRODUCTION}

Organic-inorganic perovskite solar cells have shown an unprecedented increase in power conversion efficiency, exceeding $23 \%$ over the past few years. ${ }^{1-9}$ The most common three-dimensional lead iodide perovskites have the composition $\mathrm{APbI}_{3}$, where $\mathrm{A}$ is a monovalent cation (e.g., methylammonium $\mathrm{CH}_{3} \mathrm{NH}_{3}^{+}\left(\mathrm{MA}^{+}\right)$, formamidinium $\mathrm{CH}$ $\left(\mathrm{NH}_{2}\right)_{2}{ }^{+}\left(\mathrm{FA}^{+}\right)$, or cesium $\left.\mathrm{Cs}^{+}\right)$, which fills the cuboctahedral cage formed by corner-sharing $\mathrm{PbI}_{6}$ octahedra. Various compositional engineering approaches, particularly alloying differently sized organic and inorganic cations, significantly enhance the stability of these solar cells. ${ }^{10-16}$ In this regard, the substitution of $\mathrm{Cs}^{+}$cations (up to 25\%) for the relatively larger $\mathrm{FA}^{+}$cations in $\mathrm{FAPbI}_{3}$ stabilizes the perovskite phase, increasing its resistance to chemical degradation. ${ }^{11,13-16}$ Because of their enhanced structural stability, as well as improved photovoltaic properties, these mixed-cation perovskites are currently the most promising materials for perovskite solar cells.

Recently, the application of hydrostatic pressure has emerged as an effective means of tuning the structural phases and photovoltaic properties of hybrid perovskites. ${ }^{17-23}$ Under pressure, the crystal structure of these materials evolves through several structural phases that are inaccessible by temperature variation. In addition, applied pressure reveals novel properties of halide perovskites by influencing their energetic landscapes and charge-carrier dynamics. The optimized band gap, increased charge-carrier lifetimes, reduced trap-state densities, and tuned carrier conductivities collectively result in improved photovoltaic performance in compressed halide perovskites. ${ }^{20-22}$ Solution-processed polycrystalline thin films of halide perovskites often experience significant local strain. $^{24,25}$ The experimentally observed spatial heterogeneity of the optoelectronic and photoluminescence properties of these films are speculated to be closely connected to the local strain in the perovskites. ${ }^{26-29}$ It is clear, however, that an indepth atomistic understanding of the structural and optoelectronic properties of lead halide perovskites under pressure has yet to be established.

Our recent studies have focused on defect migration, compositional engineering, and environmental stability of hybrid halide perovskites. ${ }^{30-39}$ Here we investigate the structural, optoelectronic, and defect properties of $\mathrm{FAPbI}_{3}$ and the mixed-cation $\mathrm{FA}_{0.75} \mathrm{Cs}_{0.25} \mathrm{PbI}_{3}$ under hydrostatic pressure using $a b$ initio simulation methods. Our study shows that the valence band maxima shift to a higher energy with applied pressure, indicating improved energetic alignment between the perovskite absorber and the hole collecting organic layers, which would improve solar cell performance. Conversely, under high pressure the iodide vacancies can form deep trap levels, limiting the efficiency of the cell. Our results highlight that applied pressure can be an effective tool to tune electronic landscapes and access novel optical properties in these hybrid perovskites, which would otherwise be unachievable at ambient conditions.

Received: February 14, 2019

Revised: May 13, 2019

Published: May 14, 2019 


\section{METHODS}

Density functional theory (DFT)-based $a b$ initio simulations were performed using the Vienna Ab Initio Simulation Package (VASP). ${ }^{40,41}$ The generalized gradient approximation (GGA) was considered with the Perdew-Burke-Ernzerhof functional (PBE) form. ${ }^{42}$ The plane-wave basis set, scalar relativistic pseudopotentials, and projected augmented wave $(\mathrm{PAW})^{43}$ methods were employed for all simulations. The nonlocal exchange-correlation functionals optB86b-vdW ${ }^{44}$ and a $3 \times 3 \times 3 \Gamma$-centered Monkhorst-Pack ${ }^{45}$ sampling mesh with a plane wave cutoff of $520 \mathrm{eV}$ were considered for structural optimizations, where all interatomic forces were $<0.01 \mathrm{eV}$ $\AA^{-1}$. These perovskite lattices were further optimized under static hydrostatic pressures of up to $3 \mathrm{GPa}$, which are typical experimental values. ${ }^{17-23}$ A $2 \times 2 \times 2$ (8 A cations) supercell of the parent $\mathrm{FAPbI}_{3}$ was considered for all the simulations. A $6 \times 6 \times 6 \Gamma$-centered $k$-point mesh with a Gaussian smearing of $0.01 \mathrm{eV}$ was used for Brillouin zone integrations for the electronic structure calculations. The electronic structure was further corrected by introducing spin-orbit coupling (SOC) and screened hybrid functionals, as implemented by HeydScuseria-Ernzerhof (HSE06) self-consistently. ${ }^{46}$ The HSE06 functionals include $25 \%$ of Hartree-Fock exchange with the empirical range-separation parameter $\omega=0.11 \mathrm{bohr}^{-1}$. For the band structure calculations, only SOC corrections have been considered as HSE functionals have an insignificant effect on band-edge dispersion. ${ }^{47}$ The frequency-dependent dielectric function was calculated within the random phase approximation with a $\Gamma$-centered $4 \times 4 \times 4 k$-point mesh. ${ }^{48}$ The imaginary part of this function was used to evaluate the optical response of the materials. The defect formation energies and transition levels were calculated using a $4 \times 4 \times 1$ supercell ( $16 \mathrm{~A}$ cations) while keeping all other relevant computational parameters unchanged. The Python Materials Genomics (Pymatgen) ${ }^{49}$ and Python Charged Defect Toolkit (PyCDT) $\operatorname{codes}^{50}$ libraries were used for input preparation and data analysis. Further computational details are included in the Supporting Information (section S1).

To explore the effects of external pressure at room temperature, $a b$ initio molecular dynamics simulations were performed as implemented in the $\mathrm{CP} 2 \mathrm{~K}$ package. ${ }^{51}$ To investigate structural dynamics reliably, a large cell of 64 units $(4 \times 4 \times 4$ supercell $)$ of $\mathrm{FAPbI}_{3}$ was considered. The dispersion corrections, as formulated by Grimme (DFT-D3), ${ }^{52}$ and the QUICKSTEP module ${ }^{53}$ with analytical dualspace pseudopotentials ${ }^{54}$ were employed. The Nosé-Hoover thermostat and barostat ${ }^{55}$ were used to evaluate the equilibrium dynamics under the NPT ensemble. The dynamics were performed for $40 \mathrm{ps}$ with a time step of $1 \mathrm{fs}$ where the first 5 ps was considered as the equilibration time.

\section{RESULTS AND DISCUSSION}

3.1. Structural Distortion and Dynamics. The fully optimized structure of $\mathrm{FAPbI}_{3}$ deviates from the highsymmetry cubic phase and exhibits a tetragonal distortion with an elongated $c$ lattice parameter (see section S2 of the Supporting Information for a detailed discussion). A-cation mixing in $\mathrm{FA}_{0.75} \mathrm{Cs}_{0.25} \mathrm{PbI}_{3}$ causes further tetragonal distortion due to the internal chemical pressure creating lattice strain that arises from the FA/Cs ion size mismatch. Structural modifications to the parent lattice with A-cation mixing is consistent with previous experimental and computational studies. $^{14,15,31,39}$ By investigating the mechanical properties, we find that $\mathrm{FAPbI}_{3}$ has a bulk modulus $\left(K_{0}\right)$ of $16.5 \mathrm{GPa}$, indicating a much softer lattice than typical ceramic oxide perovskites $\left(K_{0}>100\right){ }^{56}$ Notably, in $\mathrm{FA}_{0.75} \mathrm{Cs}_{0.25} \mathrm{PbI}_{3}$, the lattice stiffens with an increase of $K_{0}$ to $20 \mathrm{GPa}$. Such lattice stiffening by A-cation mixing has been found to reduce nonradiative carrier recombination in $\mathrm{MAPbI}_{3}$ doped with $\mathrm{FA}$ and guanidinium cations. ${ }^{57}$

Recent in situ synchrotron X-ray diffraction-based studies report the loss of crystallinity and amorphization of $\mathrm{FAPbI}_{3}$ under hydrostatic pressure $>4 \mathrm{GPa}^{22}$ Thus, we restrict our study to values up to $3 \mathrm{GPa}$. Under pressure, the $\mathrm{FA}_{0.75} \mathrm{Cs}_{0.25} \mathrm{PbI}_{3}$ lattice contracts in volume with an isotropic reduction in all lattice parameters, as shown in Figure 1a. This

(a)

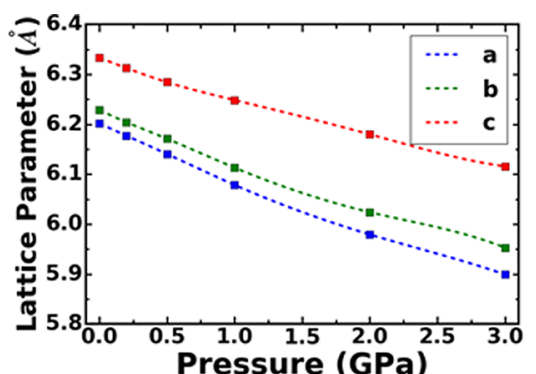

(b)

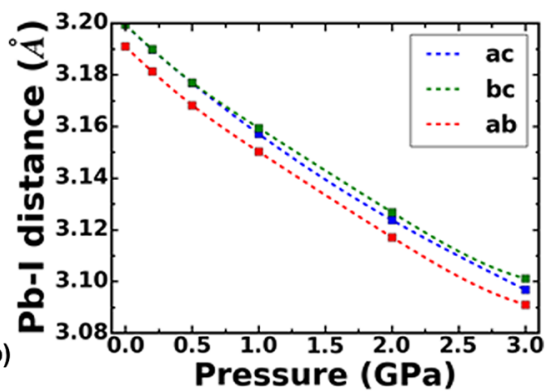

(c)

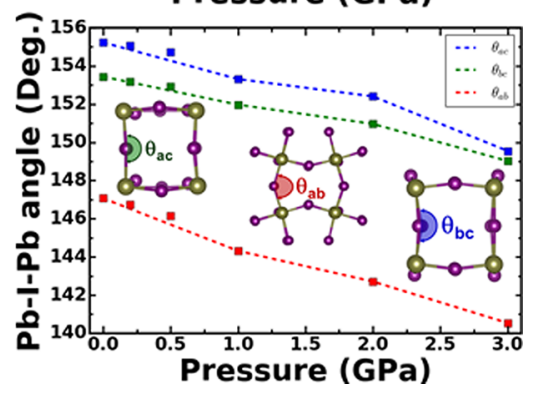

Figure 1. Pressure-induced structural distortions in $\mathrm{FA}_{0.75} \mathrm{Cs}_{0.25} \mathrm{PbI}_{3}$ : (a) lattice parameters, (b) averaged $\mathrm{Pb}-\mathrm{I}$ bond distances, and (c) $\mathrm{Pb}-\mathrm{I}-\mathrm{Pb}$ angles averaged over three Cartesian planes, with a schematic of the angles shown in the inset. Interpolated dotted lines in the plots are guides for the eye. Key: lead (brown) and iodine (pink).

contraction leads to a distortion of $\mathrm{PbI}_{6}$ octahedra through an isotropic shortening of the $\mathrm{Pb}-\mathrm{I}$ bonds in all three Cartesian planes (Figure $1 \mathrm{~b}$ ). Unlike the bond lengths, the average $\mathrm{Pb}-$ $\mathrm{I}-\mathrm{Pb}$ bond angles are anisotropic across different planes (Figure 1c). The $\mathrm{PbI}_{6}$ octahedra in the tetragonally distorted $\mathrm{FA}_{0.75} \mathrm{Cs}_{0.25} \mathrm{PbI}_{3}$ rotate primarily along the $c$-axis, and the $\mathrm{Pb}-$ $\mathrm{I}-\mathrm{Pb}$ bond angles show a maximum deviation in the $a b$-plane from $180^{\circ}$. In response to hydrostatic pressure, the octahedral tilting along the $c$-axis increases, and this consequently introduces greater anisotropic angle distortion (Figure 1c). The $\mathrm{PbI}_{6}$ octahedra adjacent to each other tilt in the same direction, showing an "in-phase" rotation. ${ }^{58}$ The anisotropic distortions of bond angles under pressure from our simulations are in good agreement with the in situ synchrotron X-ray diffraction study of $\mathrm{FAPbI}_{3}{ }^{22}$ Detailed comparisons between available experimental and computational data are given in the Supporting Information (Table S1). Therefore, the $\mathrm{PbI}_{6}$ octahedral rotation, rather than $\mathrm{Pb}-\mathrm{I}$ bond contraction, results in the internal anisotropy in these compressed perovskite structures. We note that there are limited experimental reports on pressure-induced structural changes or phase transitions of 
$\mathrm{FAPbI}_{3}$. X-ray diffraction studies of Liu et al. ${ }^{21}$ reported no evidence of a phase transition in $\mathrm{FAPbI}_{3}$ up to $7 \mathrm{GPa}$ pressure. More recent studies ${ }^{22,59}$ report phase transitions of $\mathrm{FAPbI}_{3}$ from cubic to other phases under pressure. However, the crystal systems under high pressure differ between these two studies; Wang et al. ${ }^{22}$ find cubic to orthorhombic phase (Imm2 and then $I m m m)$ transitions whereas Jiang et al. ${ }^{59}$ report tetragonal $(\mathrm{P} 4 / \mathrm{mbm})$ and cubic phases $(\operatorname{Im} \overline{3})$ at high pressures. Therefore, there is a significant degree of uncertainty and complexity regarding the exact high-pressure structures of $\mathrm{FAPbI}_{3}$. This has resulted in our initial focus on the structural and electronic properties of the observed tetragonally distorted structure as a representative model phase; we recognize that other reported crystal structures at high pressure warrant future detailed investigation.

Recent studies report strong correlation between the lattice dynamics and photovoltaic properties of these materials. ${ }^{60-62}$ To probe the effect of external pressure only, we chose to focus on the dynamics of $\mathrm{FAPbI}_{3}$. Our recent work ${ }^{31,39}$ and other studies $^{63,64}$ have investigated A-cation dynamics and their interactions with the inorganic $\mathrm{Pb} / \mathrm{I}$ frame to find their combined effect on the structural phase stability and solar cell device performance. To explore the influence of external pressure on such dynamics, we calculate the vector reorientational autocorrelation function of the cations from $a b$ initio molecular dynamics trajectories (Figure 2a). The molecular (a)
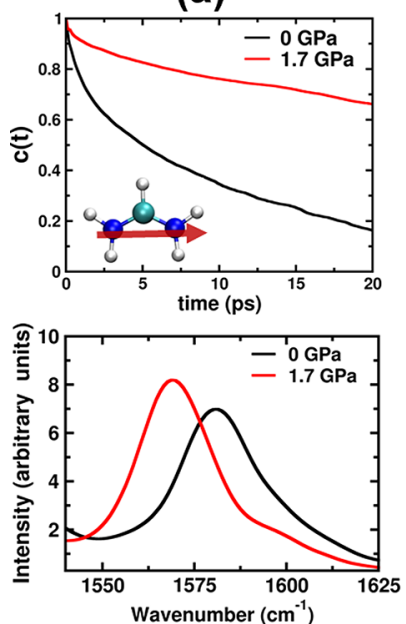

(c)
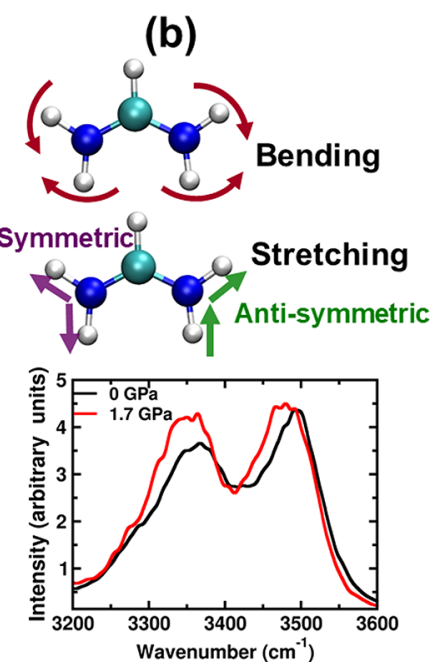

(d)
Figure 2. Cation dynamics in $\mathrm{FAPbI}_{3}$. (a) Vector autocorrelation function of $\mathrm{FA}^{+}$exhibiting the time correlation of the molecular vector (shown in the inset with a red arrow) of the cation and (b) different vibrational modes of $\mathrm{N}-\mathrm{H}$ bonds of $\mathrm{FA}^{+}$. (c) Bending and (d) symmetric and asymmetric stretching of $\mathrm{N}-\mathrm{H}$ bonds. Inset of (d) shows the shift in the vibrational peaks upon lattice compression more clearly. Key: hydrogen (white), carbon (cyan), and nitrogen (blue).

axis is defined between the two nitrogen atoms of an FA cation (inset of Figure 2a). Reorientation of the molecular axis represents a slow "jumplike" rotation of FA cations among several equilibrium conformations. ${ }^{65}$ This autocorrelation function evaluates the probability of the molecular axis remaining self-correlated over time. Thus, the fast decrease of the autocorrelation function of the $\mathrm{N}-\mathrm{N}$ molecular axis indicates a rapid reorientation of the FA cations inside the $\mathrm{Pb}$ / I frame. Several techniques, such as solid-state nuclear magnetic resonance, quasi-elastic neutron scattering, and polarization-resolved two-dimensional spectroscopy, have been used to investigate these reorientational cation dynamics. $^{65-67}$

As shown in Figure 2a, the autocorrelation function for $\mathrm{FA}^{+}$ decreases nonexponentially for $\mathrm{FAPbI}_{3}$ with and without the application of external pressure. Thus, the simulations demonstrate the dynamical coupling between the $\mathrm{FA}^{+}$cation and the inorganic lattice. Under pressure, the reorientational dynamics of the $\mathrm{FA}^{+}$are further suppressed, which is evident from the much slower decay of the function. To obtain the reorientational relaxation time of $\mathrm{FA}^{+}$molecular cations, we fit these functions with a stretched exponential decay function (see section S3 in the Supporting Information for details). For $\mathrm{FAPbI}_{3}$ at 0 and $1.7 \mathrm{GPa}$, the relaxation times for $\mathrm{FA}^{+}$are calculated as 8.8 and $88.1 \mathrm{ps}$, respectively, indicating the strong influence of external pressure on cation rotation. Raman scattering experiments have identified similar pressure-induced suppression of cation rotation in high-quality single crystals of $\mathrm{MAPbI}_{3}{ }^{68}$

Under pressure, the $\mathrm{FA}^{+}$molecules align their $\mathrm{N}-\mathrm{N}$ molecular axis preferentially along the $a$ - or $b$-axis (Figure S1). Such cation ordering in hybrid perovskites has also been found for their low-temperature phases. ${ }^{69,70}$ As discussed in previous studies on $\mathrm{FAPbI}_{3}, \mathrm{FA}^{+}$cations interact with the $\mathrm{Pb} / \mathrm{I}$ frame through $\mathrm{N}-\mathrm{H} \cdots \mathrm{I}$ hydrogen bonds. ${ }^{22,71}$ These hydrogen bonds strengthen the $\mathrm{H} \cdots \mathrm{I}$ bonds and weaken the $\mathrm{N}-\mathrm{H}$ bonds. In response, the $\mathrm{N}-\mathrm{H}$ bending and stretching vibrational modes (Figure $2 \mathrm{~b}$ ) are red-shifted in the frequency ranges $1550-1625$ and $3250-3550 \mathrm{~cm}^{-1}$, respectively (see Table S2 for all the peak values). With the compression of $\mathrm{FAPbI}_{3}$, these vibrational modes are further red-shifted in frequency, indicating a strengthening of the hydrogen bonds with applied pressure. At $1.7 \mathrm{GPa}$, the shifts are 5 and $10 \mathrm{~cm}^{-1}$ for the symmetric and asymmetric $\mathrm{N}-\mathrm{H}$ stretching modes, respectively, and $9 \mathrm{~cm}^{-1}$ for the $\mathrm{N}-\mathrm{H}$ bending mode. The simulated changes in the peak values of the vibrational frequencies with pressure match well with experimental infrared spectroscopy studies. ${ }^{22}$ The reduced volume inside the $\mathrm{Pb} / \mathrm{I}$ frame and suppressed $\mathrm{FA}^{+}$dynamics allow the amino $\mathrm{H}$ atoms to stay spatially close to the iodide sites for longer, forming stronger $\mathrm{N}-\mathrm{H} \cdots \mathrm{I}$ hydrogen-bonding interactions.

To summarize, the pressure-induced structural distortions have a major influence on the $\mathrm{FA}^{+}$cation dynamics in halide perovskites. Stronger hydrogen bonds indicate enhanced dynamical coupling between the FA cation and inorganic $\mathrm{Pb} / \mathrm{I}$ sublattices in compressed $\mathrm{FAPbI}_{3}$, which modifies the structural, electronic, and optical properties of these halide perovskites.

3.2. Tuning the Band Gap and Band Alignment. We now investigate the pressure-induced changes in the electronic properties of $\mathrm{FAPbI}_{3}$ and the mixed-cation $\mathrm{FA}_{0.75} \mathrm{Cs}_{0.25} \mathrm{PbI}_{3}$. With the application of pressure, the band gap decreases, exhibiting a red-shift in the absorption edge (Figure 3a, Figure S2, and Table S3). Thus, unlike many other semiconductors, these iodide perovskites exhibit a positive band deformation potential. We note that the underestimation of band gaps with respect to experimental values arise due to the partial consideration of many-body effects with PBE functionals, in good agreement with previous studies. ${ }^{72}$ In addition, the calculated relative red-shift in the $\mathrm{FAPbI}_{3}$ band gap using the PBE functional agrees well with experimental reports ${ }^{21}$ (see Table S3). 
(a)

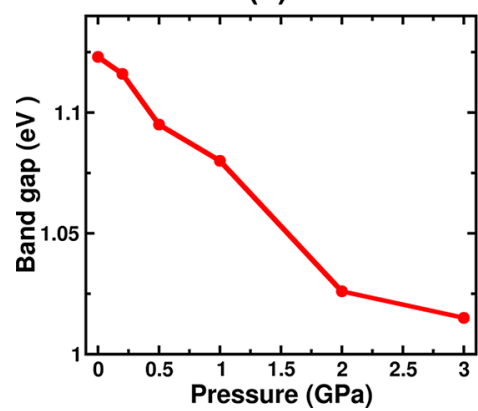

(b)

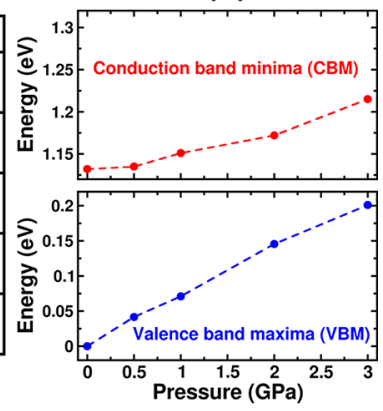

Figure 3. Change in electronic properties of $\mathrm{FA}_{0.75} \mathrm{Cs}_{0.25} \mathrm{PbI}_{3}$ with applied pressure. (a) Tuning of the band gap calculated with the HSE06 functional, including SOC corrections. (b) Modification in energy of conduction band minima (upper panel) and valence band maxima (lower panel). All band edge energies are scaled to the VBM under $0 \mathrm{GPa}$.

The alignment of the valence and conduction band edges of these perovskites with respect to the charge transport layers directly affects the extraction of photogenerated carriers from the absorber layer. ${ }^{73,74}$ Following a well-developed method for band alignment ${ }^{39,75,76}$ (as detailed in section S4 of the Supporting Information), we find that the valence band maximum (VBM) of $\mathrm{FA}_{0.75} \mathrm{Cs}_{0.25} \mathrm{PbI}_{3}$ shifts to a significantly higher energy by $\approx 0.20 \mathrm{eV}$ with an applied pressure of $3 \mathrm{GPa}$. The conduction band minimum (CBM) also moves to high energy with increased pressure, but by only $\approx 0.08 \mathrm{eV}$. The band edges of the parent $\mathrm{FAPbI}_{3}$ show similar changes with applied pressure.

To understand the variation in the band gap and band edges with pressure, we examine the partial density of states and charge densities of the VBM and CBM. As shown in Figure 4

(a)

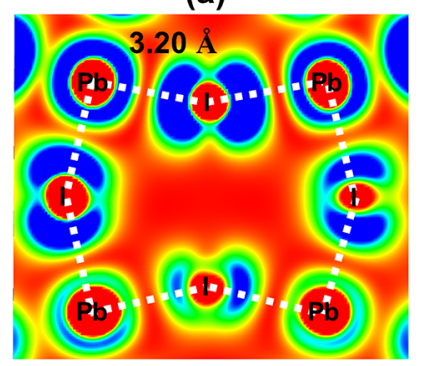

(b)

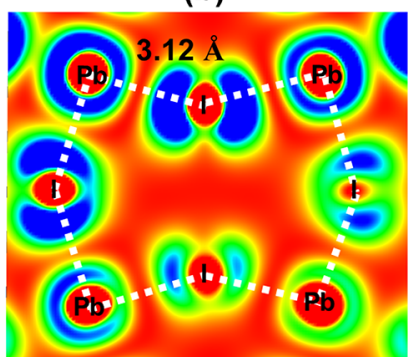

Figure 4. Electronic charge density of the VBM of $\mathrm{FA}_{0.75} \mathrm{Cs}_{0.25} \mathrm{PbI}_{3}$ under pressure of (a) 0 and (b) 2 GPa. Color code: red and blue depict 0 and $0.0001 \mathrm{e}^{-3}$, respectively. The white dashed lines indicate $\mathrm{Pb}-\mathrm{I}$ bonds, the average distance of which is given.

(and Figures S3 and S4), the antibonding overlap between $\mathrm{Pb}$ $6 s$ and I $5 p$ orbitals form the VBM, whereas nonbonding $\mathrm{Pb} 6 \mathrm{p}$ orbitals with a very small contribution from I $5 \mathrm{p}$ generate the $\mathrm{CBM}$ in $\mathrm{FA}_{0.75} \mathrm{Cs}_{0.25} \mathrm{PbI}_{3}$ and $\mathrm{FAPbI}_{3}$. With the application of pressure, the $\mathrm{Pb}-\mathrm{I}$ bond lengths shorten, and there is an increase in tilting of the $\mathrm{PbI}_{6}$ octahedra. The shorter $\mathrm{Pb}-\mathrm{I}$ bonds increase the antibonding overlap between the $\mathrm{Pb} 6 \mathrm{~s}$ and I $5 p$ orbitals, increasing the energy of the valence band edge. This enhanced interaction between participating orbitals also increases the band dispersion in the VBM. In contrast, the tilting of the $\mathrm{PbI}_{6}$ octahedra lowers the $\mathrm{Pb}-\mathrm{I}-\mathrm{Pb}$ angles, which reduces the antibonding overlap in the VBM. Thus, the VBM

energy should decrease due to increased octahedral tilting, as found in previous studies. ${ }^{39,76,77}$ However, the shorter Pb-I bonds dominate over the distorted $\mathrm{Pb}-\mathrm{I}-\mathrm{Pb}$ angles, ultimately increasing the antibonding orbital overlap between $\mathrm{Pb}$ and $\mathrm{I}$ with applied pressure. The valence band edge consequently moves to a higher energy.

With lattice compression, the band edge of the CBM also shifts to higher energy but to a smaller extent than the VBM. As the $\mathrm{Pb}-\mathrm{I}$ bonds shorten under pressure, the I $5 \mathrm{p}$ orbitals overlap with $\mathrm{Pb} 6 \mathrm{p}$ nonbonding orbitals in $\mathrm{CBM}$, increasing the covalency of the conduction band edge. The increase in covalency in the predominantly ionic conduction band results in a small upshift in its energy level. With a more covalent nature, the band dispersion of the CBM enhances under applied pressure. The charge density plots in Figure S5 further illustrate the enhanced covalent character of the conduction band under lattice compression. As the application of pressure largely shifts the VBM to a higher energy, the band gap of $\mathrm{FA}_{0.75} \mathrm{Cs}_{0.25} \mathrm{PbI}_{3}$ and $\mathrm{FAPbI}_{3}$ decreases. Our findings are in agreement with photoluminescence and ultraviolet photoelectron spectroscopy experiments. ${ }^{21,22}$

The photogenerated carriers in the absorber layer of solar cells are transferred and extracted by the energy level aligned with the charge transporting layer. ${ }^{73,74,78}$ Energetic differences between the band edge levels create an energy offset at the interface that leads to a reduction in the built-in potential, which reduces the open circuit voltage $\left(V_{o c}\right)$ and fill factor. The interface between commonly used hole transporting layers (i.e., spiro-OMeTAD and PEDOT:PSS) and halide perovskites (i.e., $\mathrm{MAPbI}_{3}, \mathrm{FAPbI}_{3}$ and their mixed phases) generally exhibit band offset energies in the range $0.2-1 \mathrm{eV}^{78} \mathrm{~A}$ reduction in this band offset energy would lead to an increase in the power conversion efficiency. Typically, the modification of the hole transporting layers by chemical doping has been explored to optimize band alignment. ${ }^{79,80}$ Interestingly, we find that the shift in the VBM of $\mathrm{FA}_{0.75} \mathrm{Cs}_{0.25} \mathrm{PbI}_{3}$ to a higher energy on applied pressure could result in an improved alignment. Thus, physical modification of perovskite layers can be an efficient alternative approach for band alignment and for optimization of the photovoltaic performance.

3.3. Spin-Splitting at Band Edges. Previous studies have demonstrated that hydrostatic pressure can be used to modify the extent of spin-splitting and the direct-indirect band transition in $\mathrm{MAPbI}_{3}{ }^{81}$ Motivated by this recent interest, we also investigate the band edge characteristics of $\mathrm{FA}_{0.75} \mathrm{Cs}_{0.25} \mathrm{PbI}_{3}$ and $\mathrm{FAPbI}_{3}$ under pressure.

As shown in Figure 5 as well as Figures S5 and S6, the CBM at the $\Gamma$ point exhibits spin-splitting for $\mathrm{FA}_{0.75} \mathrm{Cs}_{0.25} \mathrm{PbI}_{3}$ and $\mathrm{FAPbI}_{3}$ with and without external pressure. However, in the valence band, spin-splitting is insignificant and the VBM remains at the $\Gamma$ point. This different spin-splitting behavior in the band edges of $\mathrm{FA}_{0.75} \mathrm{Cs}_{0.25} \mathrm{PbI}_{3}$ results in an indirect band gap, which is narrower than the direct gap by $5-7 \mathrm{meV}$. The spin-orbit coupling strength and consequently the Rashbatype splitting directly depend on the atomic weight of the elements that form the band edges of semiconductors. Thus, spin-splitting is more prominent in the conduction band, which is dominated by heavier $\mathrm{Pb}$ atoms, than for the valence band, where relatively lighter iodine atoms largely contribute.

To quantify the magnitude of the Rashba-type effect, we calculate the Rashba parameter, $\alpha=E_{\mathrm{R}} / 2 \Delta k$, where $\Delta k$ is the SOC-induced momentum offset and $E_{\mathrm{R}}$ is the energy difference between the spin-splitted bands in a particular 


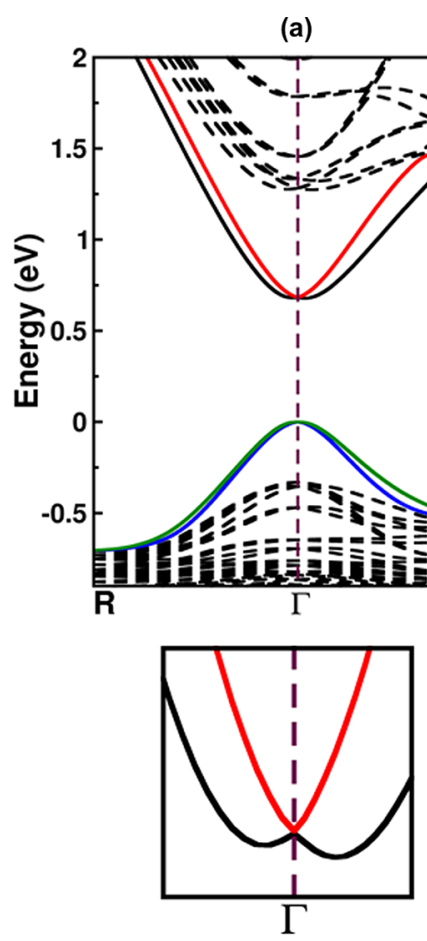

(c) (b)
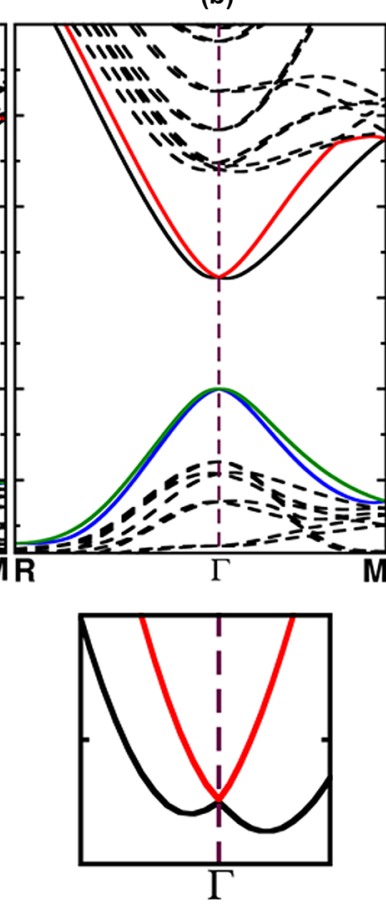

(d)
Figure 5. Rashba-type effect in mechanically compressed mixed Acation perovskites. The band structure including SOC corrections for $\mathrm{FA}_{0.75} \mathrm{Cs}_{0.25} \mathrm{PbI}_{3}$ at (a) 0 and (b) 2 GPa. The spin-splitting of the $\mathrm{CBM}$ at the $\Gamma$-point along different high-symmetry directions is evident without and with applied pressure as shown in (c) and (d), respectively. For clarity, the spin-split valence bands are represented in green and blue solid lines, and the conduction bands are shown with black and red solid lines. All bands apart from band edges are drawn by dashed black lines.

direction. By applying a hydrostatic pressure of up to $3 \mathrm{GPa}$ in $\mathrm{FA}_{0.75} \mathrm{Cs}_{0.25} \mathrm{PbI}_{3}$, we find $\alpha$ values of $0.71-0.81$ and $0.56-0.62$ $\mathrm{eV} \AA$ in the $\mathrm{M} \rightarrow \Gamma$ and $\mathrm{R} \rightarrow \Gamma$ directions, respectively (Figure S7). As shown in Figure 1, the external hydrostatic pressure distorts the mixed cation lattice anisotropically. The Rashba parameter, which is very sensitive to local structural changes in the lattice, is modified to a different extent along different highsymmetry $k$-paths. Importantly, in contrast to a previous report $^{81}$ on Rashba-type splitting in $\mathrm{MAPbI}_{3}$ decreasing with external pressure, we find this effect is slightly enhanced with lattice compression for $\mathrm{FA}_{0.75} \mathrm{Cs}_{0.25} \mathrm{PbI}_{3}$ and $\mathrm{FAPbI}_{3}$.

In the static structure, orientation of the weakly polar $\mathrm{FA}^{+}$ cations and distortion in the inorganic $\mathrm{Pb} / \mathrm{I}$ frame can break the inversion symmetry resulting in Rashba-type effects. As an atomic-scale probe of the structural origin of this effect, we replace all $\mathrm{FA}^{+}$molecules with the nonpolar $\mathrm{Cs}^{+}$in $\mathrm{FA}_{0.75} \mathrm{Cs}_{0.25} \mathrm{PbI}_{3}$ under $2 \mathrm{GPa}$ pressure and then optimize the $\mathrm{Cs}^{+}$positions only. As Figure S8a shows, the spin-splitting at the band edges of this model of $\mathrm{CsPbI}_{3}$ is very similar to that of mixed A-cation perovskites. In contrast, the high-symmetry cubic phase of $\mathrm{CsPI}_{3}$ does not exhibit spin-splitting in the band edges (see Figure S8b). Thus, the inversion symmetrybreaking of the inorganic $\mathrm{Pb} / \mathrm{I}$ frame is the cause of the Rashba-type spin-splitting in these halide perovskites and not the particular conformation of the polar FA cations. These findings demonstrate that the A cations indirectly influence the optoelectronics of hybrid perovskites by maintaining the symmetry-lowering distortions of the inorganic $\mathrm{Pb} / \mathrm{I}$ frame.

It is evident that lattice distortions in the halide perovskites modify the band gap as well as the nature of band edges and optical transitions. Interestingly, such structural distortions do not significantly enhance the spin-splitting and indirect band gap. However, as pressure can induce permanent structural distortions, it can stabilize these electronic and optical modifications for longer time scales, which can be detected experimentally.

The formation of an indirect band gap can result in the accumulation of photogenerated electrons at the spin-splitted CBM. In such a situation, the band-to-band radiative recombination of carriers can be hindered, resulting in a possible increase in carrier lifetimes. However, spin-splitting and its effect on carrier dynamics have been much debated. ${ }^{62,82,83}$

In addition to the field of photovoltaics, the Rashba-type effect in halide perovskites also opens up new applications related to spintronics and spin-orbitronics. ${ }^{84,85}$

3.4. Change in Absorption Properties. To explore the effect of pressure on the optical properties, we calculate the absorption spectra of $\mathrm{FA}_{0.75} \mathrm{Cs}_{0.25} \mathrm{PbI}_{3}$ (Figure 6a), for which
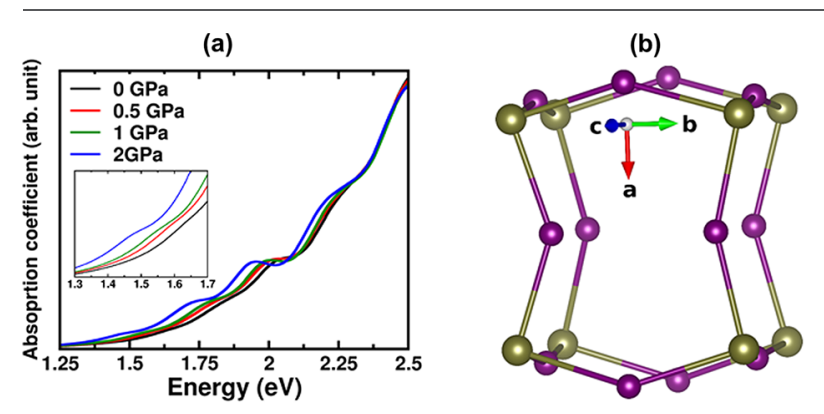

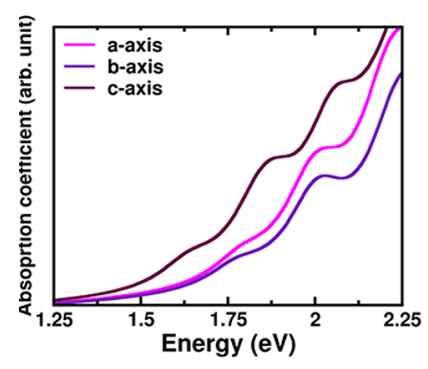

(c)

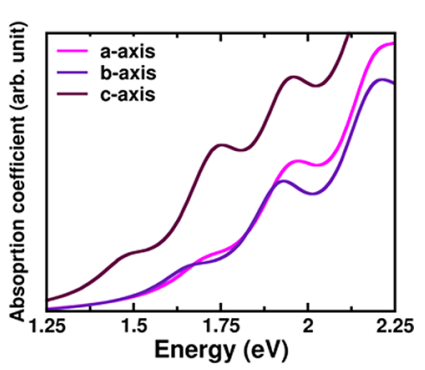

(d)
Figure 6. Optical absorption properties of $\mathrm{FA}_{0.75} \mathrm{Cs}_{0.25} \mathrm{PbI}_{3}$. (a) Spatially averaged absorption spectra under external pressure range $0-2 \mathrm{GPa}$, with inset showing the zoomed band edges. (b) Crystal axes of the anisotropic lattice. The spatially resolved absorption spectra for the crystal under (c) 0 and (d) 2 GPa pressure.

the absorption edge moves to lower energy, exhibiting a prominent red-shift in the optical spectra. As the optical transition from the VBM to CBM dominates the absorption edge, the narrowing of the electronic band gap under pressure directly causes this red-shifted absorption in these perovskites (Figure S9). ${ }^{21,22}$

In the absence of any external pressure (Figure $6 \mathrm{c}$ ), the absorption edge along the $c$-axis (shown in Figure $6 \mathrm{~b}$ ) is redshifted by 0.16 and $0.14 \mathrm{eV}$ compared to the edge along the $a$ and $b$-axes, respectively. This anisotropic character of the optical spectra is enhanced with applied pressure (Figure 6d). 
The band edge optical absorption is dominated by the transition between the VBM and CBM, with their contributions dominated by the $\mathrm{Pb} / \mathrm{I}$ frame. The $\mathrm{Pb}-\mathrm{I}$ bonds are found to be almost the same with differences of $<0.01 \AA$ in all three crystal axes (Figure $1 \mathrm{~b}$ ). In contrast, the $\mathrm{Pb}-\mathrm{I}-\mathrm{Pb}$ angles (Figure 1c) are different in all three directions due to the dominant rotation of $\mathrm{PbI}_{6}$ octahedra along the $c$-axis. The larger $\mathrm{Pb}-\mathrm{I}-\mathrm{Pb}$ angles along the $c$-axis result in the red-shifted absorption edge.

With increased pressure, the $\mathrm{PbI}_{6}$ octahedra tilt more along the $c$-axis, enhancing the anisotropy in the $\mathrm{Pb}-\mathrm{I}-\mathrm{Pb}$ angles. Consequently, the anisotropic nature of the absorption edge also becomes more prominent under pressure, as shown in Figure $6 c, d$. Thus, applying hydrostatic pressure affects the absorption properties of hybrid perovskites by changing the $\mathrm{PbI}_{6}$ octahedral tilting.

The strong anisotropic nature of the compressed lattice can account for the photoluminescence profile with additional peaks for $\mathrm{FAPbI}_{3}$ and $\mathrm{MAPbI}_{1.2} \mathrm{Br}_{1.8}{ }^{22}$ Thus, pressure can be also used to modify the excited state properties of these photovoltaic materials.

3.5. Effect on Defect States. The prevalence of intrinsic point defects (vacancies and/or interstitials) is related to their defect formation energy, which is a function of the Fermi level across the band gap (see section S1 in the Supporting Information for details). If a transition level of a defect state lies near the band edge, such that it is likely to be thermally ionized at room temperature, it is a shallow defect. Conversely, deep defects are positioned close to the middle of the band gap and are unlikely to be ionized at room temperature. While shallow trapped states do not significantly affect recombination rates, deep traps act as recombination centers for photogenerated carriers, leading to solar cell efficiency losses. ${ }^{72,86,87}$

By calculating the thermodynamic transition levels for intrinsic vacancy defects of $\mathrm{FAPbI}_{3}$ and $\mathrm{FA}_{0.75} \mathrm{Cs}_{0.25} \mathrm{PbI}_{3}$ at ambient pressure, we find that all the defects are shallow in nature, which explains the defect tolerance of hybrid perovskites (see Figure S10). ${ }^{72}$ However, under an applied pressure, the iodide vacancies $\left(\mathrm{V}_{\mathrm{I}}\right)$ change their transition levels significantly within the band gap (Figure 7). We also see a significant shift of the $\mathrm{V}_{\mathrm{Pb}}$ doubly charged transition states at $2 \mathrm{GPa}$, although this shift moves the transition states to within the valence band and therefore would not affect recombination rates (Figure S10). Moreover, according to Shockley-ReadHall recombination theory only neutral and singly charged defects act as recombination centers. ${ }^{88,89}$ We therefore focused on the defect properties of iodide vacancies $\left(V_{I}\right)$ in their positive, neutral, and negatively charged states.

As pressure introduces large structural distortions leading to nonequivalent iodide sites, we have calculated transition levels considering all such sites and find very similar defect properties. Consistent with other computational studies on halide perovskites at ambient pressure, iodide vacancies are stabilized mainly in their positively and negatively charged states, with $\epsilon(+/ 0)$ and $\epsilon(0 /-)$ transitions near or in the CBM (Figure $7 \mathrm{a}){ }^{72,90,91}$ At low pressures $(\leq 0.5 \mathrm{GPa})$, the transition state levels of vacancy defects remain largely unchanged and would not significantly affect the charge carrier lifetime (Figure 7a and Figure S11).

At higher pressures (2 GPa) (Figure $7 \mathrm{~b}$ and Figure S12), deep-level states appear with the $\epsilon(+/ 0)$ transition at $\sim 0.25-$ $0.65 \mathrm{eV}$ from the CBM. The calculated optical spectra consequently exhibits prominent absorption peaks below the (a)

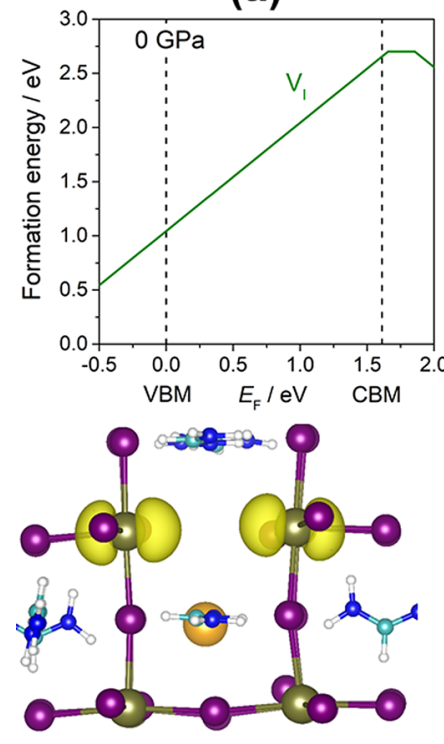

(c) (b)
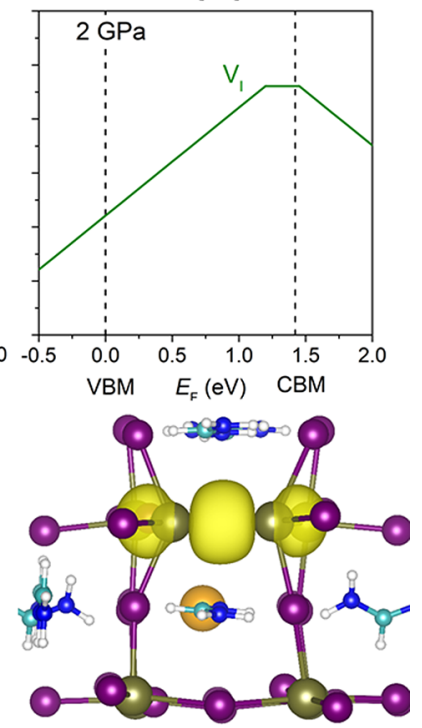

(d)
Figure 7. Iodide vacancy formation energies in $\mathrm{FA}_{0.75} \mathrm{Cs}_{0.25} \mathrm{PbI}_{3}$ at the lowest energy equatorial sites. The most stable charge state is shown at (a) 0 and (b) $2 \mathrm{GPa}$. The charge densities of the defect state at these pressures have been shown in (c) and (d). The stable charge states at other pressures are shown in the Supporting Information.

band gap energy (Figure S13). Formation of such midgap states would lead to higher recombination rates that are detrimental to solar cell performance. A recent study by Jones et $\mathrm{al}^{24}$ shows that grain clusters of $\mathrm{MAPbI}_{3}$ under a compressive strain have shorter photoluminescence lifetimes and carrier dynamics compared to unstrained regions. This is in agreement with our work, where we show a shift of shallow to deep states of $\mathrm{V}_{\mathrm{I}}$ in $\mathrm{FAPbI}_{3}$ and $\mathrm{FA}_{0.75} \mathrm{Cs}_{0.25} \mathrm{PbI}_{3}$, which would lead to a reduction in charge carrier lifetimes.

To understand the origin of these deep-level states, the structure and electronic nature of the defect sites have been explored. As shown in Figure $7 \mathrm{c}, \mathrm{d}$, the two $\mathrm{Pb}$ atoms nearest to the $\mathrm{V}_{\mathrm{I}}$ are $\sim 6.1 \AA$ apart at $0 \mathrm{GPa}$ pressure but form $\mathrm{Pb}-\mathrm{Pb}$ dimers that are $\sim 4.2 \AA$ apart at $2 \mathrm{GPa}$. Hence, applied pressure pushes these two $\mathrm{Pb}$ atoms together to form bonds across the $\mathrm{V}_{\mathrm{I}}$ site, which shifts down the corresponding energy level, placing it deep within the band gap. The charge densities of the defect states clearly show localization over these $\mathrm{Pb}-\mathrm{Pb}$ dimers. Such midgap states with strongly localized charge density give rise to deep-level states.

\section{CONCLUSIONS}

The effects of external pressure on the structural and optoelectronic properties of iodide perovskites are thoroughly explored at the atomic level. By combining static and dynamic $a b$ initio computations, we have investigated $\mathrm{FAPbI}_{3}$ and mixed-cation $\mathrm{FA}_{0.75} \mathrm{Cs}_{0.25} \mathrm{PbI}_{3}$, revealing the following key conclusions:

(a) With applied pressure, the inorganic $\mathrm{Pb} / \mathrm{I}$ framework undergoes structural distortion with a reduction in the $\mathrm{Pb}-\mathrm{I}$ bond lengths and increased tilting of the corner-sharing $\mathrm{PbI}_{6}$ octahedra. These structural distortions restrict the rotational dynamics of the $\mathrm{FA}^{+}$molecular cation and enhance its coupling with the $\mathrm{Pb} / \mathrm{I}$ inorganic framework with stronger $\mathrm{N}-\mathrm{H} \cdots \mathrm{I}$ hydrogen bonding. 
(b) The electronic structure responds strongly to the lattice compression with significant narrowing of the band gap. The valence band maximum shifts to higher energy with applied pressure, indicating improved energy level alignment between the perovskite absorber and hole collecting organic layers, which may enhance solar cell performance.

(c) Symmetry breaking in the compressed lattice leads to Rashba-type spin-splitting of the conduction band. The absorption coefficients exhibit strong anisotropy along $a, b$, and $c$ lattice directions due to the pressure-induced distorted structure.

(d) The intrinsic vacancy defects of $\mathrm{FAPbI}_{3}$ and $\mathrm{FA}_{0.75} \mathrm{Cs}_{0.25} \mathrm{PbI}_{3}$ at ambient pressure are all shallow in nature, which explains the defect tolerance of hybrid perovskites. Under pressure, the iodide vacancy is modified to a deep-level state that would strongly affect the optoelectronic properties of compressed lattices.

Our study provides an atomistic understanding of pressureinduced effects as a materials design strategy to tune the structure-property relationships of lead iodide perovskites.

\section{ASSOCIATED CONTENT}

\section{S Supporting Information}

The Supporting Information is available free of charge on the ACS Publications website at DOI: 10.1021/acs.chemmater.9b00648.

Detailed methods for defect calculations, $\mathrm{FAPbI}_{3}$ structure under pressure, reorientation relaxation time, band alignment, detailed optoelectronic and defect properties (PDF)

\section{AUTHOR INFORMATION}

\section{Corresponding Authors}

*E-mail: dibyajnc@gmail.com.

*E-mail: m.s.islam@bath.ac.uk.

\section{ORCID $\odot$}

Dibyajyoti Ghosh: 0000-0002-3640-7537

James A. Dawson: 0000-0002-3946-5337

M. Saiful Islam: 0000-0003-3882-0285

\section{Notes}

The authors declare no competing financial interest.

\section{ACKNOWLEDGMENTS}

This work was supported by the Energy oriented Centre of Excellence (EoCoE), Grant Agreement No. 676629, funded within the Horizon2020 framework of the European Union. M.S.I. and D.G. acknowledge support from the UK EPSRC Energy Materials Programme Grant (EP/K016288/1) and Archer HPC facilities through the Materials Chemistry Consortium (EP/L000202).

\section{REFERENCES}

(1) Kojima, A.; Teshima, K.; Shirai, Y.; Miyasaka, T. Organometal Halide Perovskites as Visible-light Sensitizers for Photovoltaic Cells. J. Am. Chem. Soc. 2009, 131, 6050-6051.

(2) Lee, M. M.; Teuscher, J.; Miyasaka, T.; Murakami, T. N.; Snaith, H. J. Efficient Hybrid Solar Cells Based on Meso-superstructured Organometal Halide Perovskites. Science 2012, 338, 643-647.

(3) Green, M. A.; Ho-Baillie, A.; Snaith, H. J. The Emergence of Perovskite Solar Cells. Nat. Photonics 2014, 8, 506-514.

(4) Grätzel, M. The Light and Shade of Perovskite Solar Cells. Nat. Mater. 2014, 13, 838-842.
(5) Chen, W.; Wu, Y.; Yue, Y.; Liu, J.; Zhang, W.; Yang, X.; Chen, H.; Bi, E.; Ashraful, I.; Grätzel, M.; Han, L. Efficient and Stable Largearea Perovskite Solar Cells with Inorganic Charge Extraction Layers. Science 2015, 350, 944-948.

(6) Yang, W. S.; Noh, J. H.; Jeon, N. J.; Kim, Y. C.; Ryu, S.; Seo, J.; Seok, S. I. High-performance Photovoltaic Perovskite Layers Fabricated Through Intramolecular Exchange. Science 2015, 348, 1234-1237.

(7) Egger, D. A.; Rappe, A. M.; Kronik, L. Hybrid OrganicInorganic Perovskites on the Move. Acc. Chem. Res. 2016, 49, 573581.

(8) Correa-Baena, J.-P.; Abate, A.; Saliba, M.; Tress, W.; Jacobsson, T. J.; Grätzel, M.; Hagfeldt, A. The Rapid Evolution of Highly Efficient Perovskite Solar Cells. Energy Environ. Sci. 2017, 10, 710727.

(9) Egger, D. A.; Bera, A.; Cahen, D.; Hodes, G.; Kirchartz, T.; Kronik, L.; Lovrincic, R.; Rappe, A. M.; Reichman, D. R.; Yaffe, O. What Remains Unexplained about the Properties of Halide Perovskites? Adv. Mater. 2018, 30, 1800691.

(10) Saliba, M.; Matsui, T.; Seo, J.-Y.; Domanski, K.; Correa-Baena, J.-P.; Nazeeruddin, M. K.; Zakeeruddin, S. M.; Tress, W.; Abate, A.; Hagfeldt, A.; Grätzel, M. Cesium-containing Triple Cation Perovskite Solar Cells: Improved Stability, Reproducibility and High Efficiency. Energy Environ. Sci. 2016, 9, 1989-1997.

(11) McMeekin, D. P.; Sadoughi, G.; Rehman, W.; Eperon, G. E.; Saliba, M.; Hörantner, M. T.; Haghighirad, A.; Sakai, N.; Korte, L.; Rech, B.; Johnston, M. B.; Herz, L. M.; Snaith, H. J. A Mixed-cation Lead Mixed-halide Perovskite Absorber for Tandem Solar Cells. Science 2016, 351, 151-155.

(12) Niemann, R. G.; Gouda, L.; Hu, J.; Tirosh, S.; Gottesman, R.; Cameron, P. J.; Zaban, A. $\mathrm{Cs}^{+}$incorporation into $\mathrm{CH}_{3} \mathrm{NH}_{3} \mathrm{PbI}_{3}$ perovskite: substitution limit and stability enhancement. J. Mater. Chem. A 2016, 4, 17819-17827.

(13) Rehman, W.; McMeekin, D. P.; Patel, J. B.; Milot, R. L.; Johnston, M. B.; Snaith, H. J.; Herz, L. M. Photovoltaic Mixed-cation Lead Mixed-halide Perovskites: Links between Crystallinity, Photostability and Electronic properties. Energy Environ. Sci. 2017, 10, 361369.

(14) Lee, J.-W.; Kim, D.-H.; Kim, H.-S.; Seo, S.-W.; Cho, S. M.; Park, N.-G. Formamidinium and Cesium Hybridization for Photo-and Moisture-stable Perovskite Solar Cell. Adv. Energy Mater. 2015, 5, 1501310.

(15) Prasanna, R.; Gold-Parker, A.; Leijtens, T.; Conings, B.; Babayigit, A.; Boyen, H.-G.; Toney, M. F.; McGehee, M. D. Band Gap Tuning via Lattice Contraction and Octahedral Tilting in Perovskite Materials for Photovoltaics. J. Am. Chem. Soc. 2017, 139, 1111711124.

(16) Turren-Cruz, S.-H.; Hagfeldt, A.; Saliba, M. Methylammoniumfree, high-performance and stable perovskite solar cells on a planar architecture. Science 2018, 362, 449-453.

(17) Jaffe, A.; Lin, Y.; Karunadasa, H. I. Halide Perovskites Under Pressure: Accessing New Properties Through Lattice Compression. ACS Energy Lett. 2017, 2, 1549-1555.

(18) Jaffe, A.; Lin, Y.; Beavers, C. M.; Voss, J.; Mao, W. L.; Karunadasa, H. I. High-pressure single-crystal structures of 3D leadhalide hybrid perovskites and pressure effects on their electronic and optical properties. ACS Cent. Sci. 2016, 2, 201-209.

(19) Jiang, S.; Fang, Y.; Li, R.; Xiao, H.; Crowley, J.; Wang, C.; White, T. J.; Goddard, W. A.; Wang, Z.; Baikie, T.; Fang, J. PressureDependent Polymorphism and Band-Gap Tuning of Methylammonium Lead Iodide Perovskite. Angew. Chem., Int. Ed. 2016, 55, 65406544.

(20) Kong, L.; Liu, G.; Gong, J.; Hu, Q.; Schaller, R. D.; Dera, P.; Zhang, D.; Liu, Z.; Yang, W.; Zhu, K.; et al. Simultaneous band-gap narrowing and carrier-lifetime prolongation of organic-inorganic trihalide perovskites. Proc. Natl. Acad. Sci. U. S. A. 2016, 113, 89108915.

(21) Liu, G.; Kong, L.; Gong, J.; Yang, W.; Mao, H.-k.; Hu, Q.; Liu, Z.; Schaller, R. D.; Zhang, D.; Xu, T. Pressure-Induced Bandgap 
Optimization in Lead-Based Perovskites with Prolonged Carrier Lifetime and Ambient Retainability. Adv. Funct. Mater. 2017, 27, 1604208.

(22) Wang, P.; Guan, J.; Galeschuk, D. T.; Yao, Y.; He, C. F.; Jiang, S.; Zhang, S.; Liu, Y.; Jin, M.; Jin, C.; Song, Y. Pressure-Induced Polymorphic, Optical, and Electronic Transitions of Formamidinium Lead Iodide Perovskite. J. Phys. Chem. Lett. 2017, 8, 2119-2125.

(23) Postorino, P.; Malavasi, L. Pressure-induced effects in organicinorganic hybrid perovskites. J. Phys. Chem. Lett. 2017, 8, 2613-2622.

(24) Jones, T. W.; Osherov, A.; Alsari, M.; Sponseller, M.; Duck, B. C.; Jung, Y.-K.; Settens, C.; Niroui, F.; Brenes, R.; Stan, C. V.; et al. Local Strain Heterogeneity Influences the Optoelectronic Properties of Halide Perovskites. Energy Environ. Sci. 2019, 12, 596.

(25) Zhao, J.; Deng, Y.; Wei, H.; Zheng, X.; Yu, Z.; Shao, Y.; Shield, J. E.; Huang, J. Strained hybrid perovskite thin films and their impact on the intrinsic stability of perovskite solar cells. Sci. Adv. 2017, 3, eaao5616.

(26) de Quilettes, D. W.; Vorpahl, S. M.; Stranks, S. D.; Nagaoka, H.; Eperon, G. E.; Ziffer, M. E.; Snaith, H. J.; Ginger, D. S. Impact of microstructure on local carrier lifetime in perovskite solar cells. Science 2015, 348, 683-686.

(27) Guo, Z.; Manser, J. S.; Wan, Y.; Kamat, P. V.; Huang, L. Spatial and temporal imaging of long-range charge transport in perovskite thin films by ultrafast microscopy. Nat. Commun. 2015, 6, 7471.

(28) Leblebici, S. Y.; Leppert, L.; Li, Y.; Reyes-Lillo, S. E.; Wickenburg, S.; Wong, E.; Lee, J.; Melli, M.; Ziegler, D.; Angell, D. $\mathrm{K}$; et al. Facet-dependent photovoltaic efficiency variations in single grains of hybrid halide perovskite. Nat. Energy. 2016, 1, 16093.

(29) deQuilettes, D. W.; Jariwala, S.; Burke, S.; Ziffer, M. E.; Wang, J. T.-W.; Snaith, H. J.; Ginger, D. S. Tracking Photoexcited Carriers in Hybrid Perovskite Semiconductors: Trap-Dominated Spatial Heterogeneity and Diffusion. ACS Nano 2017, 11, 11488-11496.

(30) Eames, C.; Frost, J. M.; Barnes, P. R.; O'regan, B. C.; Walsh, A.; Islam, M. S. Ionic Transport in Hybrid Lead Iodide Perovskite Solar Cells. Nat. Commun. 2015, 6, 7497.

(31) Ghosh, D.; Walsh Atkins, P.; Islam, M. S.; Walker, A. B.; Eames, C. Good Vibrations: Locking of Octahedral Tilting in MixedCation Iodide Perovskites for Solar Cells. ACS Energy Lett. 2017, 2, 2424-2429.

(32) Aristidou, N.; Eames, C.; Sanchez-Molina, I.; Bu, X.; Kosco, J.; Islam, M. S.; Haque, S. A. Fast Oxygen Diffusion and Iodide Defects Mediate Oxygen-induced Degradation of Perovskite Solar Cells. Nat. Commun. 2017, 8, 15218.

(33) Charles, B.; Dillon, J.; Weber, O. J.; Islam, M. S.; Weller, M. T. Understanding the stability of mixed A-cation lead iodide perovskites. J. Mater. Chem. A 2017, 5, 22495-22499.

(34) Nagane, S.; Ghosh, D.; Hoye, R. L.; Zhao, B.; Ahmad, S.; Walker, A. B.; Islam, M. S.; Ogale, S.; Sadhanala, A. Lead-Free Perovskite Semiconductors Based on Germanium-Tin Solid Solutions: Structural and Optoelectronic Properties. J. Phys. Chem. C 2018, 122, 5940-5947.

(35) Pering, S. R.; Deng, W.; Troughton, J. R.; Kubiak, P.; Ghosh, D.; Niemann, R.; Brivio, F.; Jeffrey, F.; Walker, A.; Islam, M.; et al. Azetidinium lead iodide for perovskite solar cells. J. Mater. Chem. A 2017, 5, 20658-20665.

(36) Brenes, R.; Eames, C.; Bulović, V.; Islam, M. S.; Stranks, S. D. The Impact of Atmosphere on the Local Luminescence Properties of Metal Halide Perovskite Grains. Adv. Mater. 2018, 30, 1706208.

(37) Brenes, R.; Guo, D.; Osherov, A.; Noel, N. K.; Eames, C.; Hutter, E. M.; Pathak, S. K.; Niroui, F.; Friend, R. H.; Islam, M. S. A.; et al. Metal Halide Perovskite Polycrystalline Films Exhibiting Properties of Single Crystals. Joule 2017, 1, 155-167.

(38) Dawson, J. A.; Naylor, A. J.; Eames, C.; Roberts, M.; Zhang, W.; Snaith, H. J.; Bruce, P. G.; Islam, M. S. Mechanisms of lithium intercalation and conversion processes in organic-inorganic halide perovskites. ACS Energy Lett. 2017, 2, 1818-1824.

(39) Ghosh, D.; Smith, A. R.; Walker, A. B.; Islam, M. S. Mixed ACation Perovskites for Solar Cells: Atomic-Scale Insights Into
Structural Distortion, Hydrogen Bonding, and Electronic Properties. Chem. Mater. 2018, 30, 5194-5204.

(40) Kresse, G.; Hafner, J. Ab initio molecular dynamics for liquid metals. Phys. Rev. B: Condens. Matter Mater. Phys. 1993, 47, 558.

(41) Kresse, G.; Hafner, J. Ab initio molecular-dynamics simulation of the liquid-metal-amorphous-semiconductor transition in germanium. Phys. Rev. B: Condens. Matter Mater. Phys. 1994, 49, 14251.

(42) Perdew, J. P.; Burke, K.; Ernzerhof, M. Generalized Gradient Approximation Made Simple. Phys. Rev. Lett. 1996, 77, 3865.

(43) Kresse, G.; Joubert, D. From ultrasoft pseudopotentials to the projector augmented-wave method. Phys. Rev. B: Condens. Matter Mater. Phys. 1999, 59, 1758.

(44) Klimeš, J.; Bowler, D. R.; Michaelides, A. Van der Waals density functionals applied to solids. Phys. Rev. B: Condens. Matter Mater. Phys. 2011, 83, 195131.

(45) Monkhorst, H. J.; Pack, J. D. Special points for Brillouin-zone integrations. Phys. Rev. B 1976, 13, 5188.

(46) Heyd, J.; Scuseria, G. E.; Ernzerhof, M. Hybrid functionals based on a screened Coulomb potential. J. Chem. Phys. 2003, 118, $8207-8215$.

(47) Leppert, L.; Reyes-Lillo, S. E.; Neaton, J. B. Electric field-and strain-induced Rashba effect in hybrid halide perovskites. J. Phys. Chem. Lett. 2016, 7, 3683-3689.

(48) Gajdoš, M.; Hummer, K.; Kresse, G.; Furthmüller, J.; Bechstedt, F. Linear optical properties in the projector-augmented wave methodology. Phys. Rev. B: Condens. Matter Mater. Phys. 2006, 73,045112

(49) Ong, S. P.; Richards, W. D.; Jain, A.; Hautier, G.; Kocher, M.; Cholia, S.; Gunter, D.; Chevrier, V.; Persson, K. A.; Ceder, G. Python Materials Genomics (pymatgen): A robust, open-source python library for materials analysis. Comput. Mater. Sci. 2013, 68, 314-319.

(50) Broberg, D.; Medasani, B.; Zimmermann, N. E. R.; Yu, G. D.; Canning, A.; Haranczyk, M.; Asta, M.; Hautier, G. PyCDT: A Python toolkit for modeling point defects in semiconductors and insulators. Comput. Phys. Commun. 2018, 226, 165-179.

(51) Hutter, J.; Iannuzzi, M.; Schiffmann, F.; VandeVondele, J. CP2K: Atomistic Simulations of Condensed Matter Systems. Wiley Interdiscip. Rev. Comput. Mol. Sci. 2014, 4, 15-25.

(52) Grimme, S. Semiempirical GGA-type Density Functional Constructed with a Long-range Dispersion Correction. J. Comput. Chem. 2006, 27, 1787-1799.

(53) VandeVondele, J.; Krack, M.; Mohamed, F.; Parrinello, M.; Chassaing, T.; Hutter, J. Quickstep: Fast and Accurate Density Functional Calculations using a Mixed Gaussian and Plane Waves Approach. Comput. Phys. Commun. 2005, 167, 103-128.

(54) Hartwigsen, C.; Gœdecker, S.; Hutter, J. Relativistic separable dual-space Gaussian pseudopotentials from $\mathrm{H}$ to Rn. Phys. Rev. B: Condens. Matter Mater. Phys. 1998, 58, 3641.

(55) Martyna, G. J.; Tobias, D. J.; Klein, M. L. Constant Pressure Molecular Dynamics Algorithms. J. Chem. Phys. 1994, 101, 41774189.

(56) Mattern, E.; Matas, J.; Ricard, Y.; Bass, J. Lower mantle composition and temperature from mineral physics and thermodynamic modelling. Geophys. J. Int. 2005, 160, 973-990.

(57) He, J.; Fang, W.-H.; Long, R.; Prezhdo, O. V. Increased Lattice Stiffness Suppresses Nonradiative Charge Recombination in MAPbI3 Doped with Larger Cations: Time-Domain Ab Initio Analysis. ACS Energy Lett. 2018, 3, 2070-2076.

(58) Glazer, A. The Classification of Tilted Octahedra in Perovskites. Acta Crystallogr., Sect. B: Struct. Crystallogr. Cryst. Chem. 1972, 28, 3384-3392.

(59) Jiang, S.; Luan, Y.; Jang, J. I.; Baikie, T.; Huang, X.; Li, R.; Saouma, F. O.; Wang, Z.; White, T. J.; Fang, J. Phase Transitions of Formamidinium Lead Iodide Perovskite under Pressure. J. Am. Chem. Soc. 2018, 140, 13952-13957.

(60) Stroppa, A.; Di Sante, D.; Barone, P.; Bokdam, M.; Kresse, G.; Franchini, C.; Whangbo, M.-H.; Picozzi, S. Tunable ferroelectric polarization and its interplay with spin-orbit coupling in tin iodide perovskites. Nat. Commun. 2014, 5, 5900. 
(61) Yaffe, O.; Guo, Y.; Tan, L. Z.; Egger, D. A.; Hull, T.; Stoumpos, C. C.; Zheng, F.; Heinz, T. F.; Kronik, L.; G, K. M.; et al. Local polar fluctuations in lead halide perovskite crystals. Phys. Rev. Lett. 2017, $118,136001$.

(62) Niesner, D.; Hauck, M.; Shrestha, S.; Levchuk, I.; Matt, G. J.; Osvet, A.; Batentschuk, M.; Brabec, C.; Weber, H. B.; Fauster, T. Structural fluctuations cause spin-split states in tetragonal (CH3NH3) $\mathrm{PbI} 3$ as evidenced by the circular photogalvanic effect. Proc. Natl. Acad. Sci. U. S. A. 2018, 115, 9509-9514.

(63) Gallop, N. P.; Selig, O.; Giubertoni, G.; Bakker, H. J.; Rezus, Y. L.; Frost, J. M.; Jansen, T. L.; Lovrincic, R.; Bakulin, A. A. Rotational Cation Dynamics in Metal-Halide Perovskites: Effect on Phonons and Material Properties. J. Phys. Chem. Lett. 2018, 9, 5987-5997.

(64) Kang, B.; Biswas, K. Preferential $\mathrm{CH}_{3} \mathrm{NH}_{3}{ }^{+}$Alignment and Octahedral Tilting Affect Charge Localization in Cubic Phase $\mathrm{CH}_{3} \mathrm{NH}_{3} \mathrm{PbI}_{3}$. J. Phys. Chem. C 2017, 121, 8319-8326.

(65) Bakulin, A. A.; Selig, O.; Bakker, H. J.; Rezus, Y. L.; Müller, C.; Glaser, T.; Lovrincic, R.; Sun, Z.; Chen, Z.; Walsh, A.; Frost, J. M.; Jansen, T. L. C. Real-time observation of organic cation reorientation in methylammonium lead iodide perovskites. J. Phys. Chem. Lett. 2015, 6, 3663-3669.

(66) Fabini, D. H.; Siaw, T. A.; Stoumpos, C. C.; Laurita, G.; Olds, D.; Page, K.; Hu, J. G.; Kanatzidis, M. G.; Han, S.; Seshadri, R. Universal Dynamics of Molecular Reorientation in Hybrid Lead Iodide Perovskites. J. Am. Chem. Soc. 2017, 139, 16875-16884.

(67) Chen, T.; Foley, B. J.; Ipek, B.; Tyagi, M.; Copley, J. R.; Brown, C. M.; Choi, J. J.; Lee, S.-H. Rotational dynamics of organic cations in the $\mathrm{CH}_{3} \mathrm{NH}_{3} \mathrm{PbI}_{3}$ perovskite. Phys. Chem. Chem. Phys. 2015, 17, 31278-31286.

(68) Francisco-López, A.; Charles, B.; Weber, O. J.; Alonso, M. I.; Garriga, M.; Campoy-Quiles, M.; Weller, M. T.; Goñi, A. R. PressureInduced Locking of Methylammonium Cations versus Amorphization in Hybrid Lead Iodide Perovskites. J. Phys. Chem. C 2018, 122, 22073-22082.

(69) Weber, O. J.; Ghosh, D.; Gaines, S.; Henry, P. F.; Walker, A. B.; Islam, M. S.; Weller, M. T. Phase behavior and polymorphism of formamidinium lead iodide. Chem. Mater. 2018, 30, 3768-3778.

(70) Weller, M. T.; Weber, O. J.; Henry, P. F.; Di Pumpo, A. M.; Hansen, T. C. Complete Structure and Cation Orientation in the Perovskite Photovoltaic Methylammonium Lead Iodide between 100 and 352 K. Chem. Commun. 2015, 51, 4180-4183.

(71) Weller, M. T.; Weber, O. J.; Frost, J. M.; Walsh, A. Cubic perovskite structure of black formamidinium lead iodide, $\alpha$-[HC$\left.\left(\mathrm{NH}_{2}\right)_{2}\right] \mathrm{PbI}_{3}$, at 298 K. J. Phys. Chem. Lett. 2015, 6, 3209-3212.

(72) Meggiolaro, D.; De Angelis, F. First-Principles Modeling of Defects in Lead Halide Perovskites: Best Practices and Open Issues. ACS Energy Lett. 2018, 3, 2206-2222.

(73) Baena, J. P. C.; Steier, L.; Tress, W.; Saliba, M.; Neutzner, S.; Matsui, T.; Giordano, F.; Jacobsson, T. J.; Kandada, A. R. S.; et al. Highly efficient planar perovskite solar cells through band alignment engineering. Energy Environ. Sci. 2015, 8, 2928-2934.

(74) Yin, W.-J.; Yang, J.-H.; Kang, J.; Yan, Y.; Wei, S.-H. Halide perovskite materials for solar cells: a theoretical review. J. Mater. Chem. A 2015, 3, 8926-8942.

(75) Yi, C.; Luo, J.; Meloni, S.; Boziki, A.; Ashari-Astani, N.; Grätzel, C.; Zakeeruddin, S. M.; Röthlisberger, U.; Grätzel, M. Entropic Stabilization of Mixed A-cation $\mathrm{ABX}_{3}$ Metal Halide Perovskites for High Performance Perovskite Solar Cells. Energy Environ. Sci. 2016, 9, 656-662.

(76) Meloni, S.; Palermo, G.; Ashari-Astani, N.; Grätzel, M.; Rothlisberger, U. Valence and conduction band tuning in halide perovskites for solar cell applications. J. Mater. Chem. A 2016, 4, 15997-16002.

(77) Lee, J.-H.; Bristowe, N. C.; Lee, J. H.; Lee, S.-H.; Bristowe, P. D.; Cheetham, A. K.; Jang, H. M. Resolving the Physical Origin of Octahedral Tilting in Halide Perovskites. Chem. Mater. 2016, 28, 4259-4266.
(78) Kim, H.; Lim, K.-G.; Lee, T.-W. Planar heterojunction organometal halide perovskite solar cells: roles of interfacial layers. Energy Environ. Sci. 2016, 9, 12-30.

(79) Lim, K.-G.; Kim, H.-B.; Jeong, J.; Kim, H.; Kim, J. Y.; Lee, T.$\mathrm{W}$. Boosting the power conversion efficiency of perovskite solar cells using self-organized polymeric hole extraction layers with high work function. Adv. Mater. 2014, 26, 6461-6466.

(80) Lin, Q.; Armin, A.; Nagiri, R. C. R.; Burn, P. L.; Meredith, P. Electro-optics of perovskite solar cells. Nat. Photonics 2015, 9, 106112.

(81) Wang, T.; Daiber, B.; Frost, J. M.; Mann, S. A.; Garnett, E. C.; Walsh, A.; Ehrler, B. Indirect to direct bandgap transition in methylammonium lead halide perovskite. Energy Environ. Sci. 2017, 10, 509-515.

(82) Frohna, K.; Deshpande, T.; Harter, J.; Peng, W.; Barker, B. A.; Neaton, J. B.; Louie, S. G.; Bakr, O. M.; Hsieh, D.; Bernardi, M. Inversion symmetry and bulk Rashba effect in methylammonium lead iodide perovskite single crystals. Nat. Commun. 2018, 9, 1829.

(83) Herz, L. M. Charge-carrier mobilities in metal halide perovskites: Fundamental mechanisms and limits. ACS Energy Lett. 2017, 2, 1539-1548.

(84) Stranks, S. D.; Plochocka, P. The influence of the Rashba effect. Nat. Mater. 2018, 17, 381-382.

(85) Kepenekian, M.; Even, J. Rashba and Dresselhaus Couplings in Halide Perovskites: Accomplishments and Opportunities for Spintronics and Spin-Orbitronics. J. Phys. Chem. Lett. 2017, 8, 33623370.

(86) Shockley, W.; Read, W. T. Statistics of the Recombinations of Holes and Electrons. Phys. Rev. 1952, 87, 835-842.

(87) Liu, N.; Yam, C. First-principles study of intrinsic defects in formamidinium lead triiodide perovskite solar cell absorbers. Phys. Chem. Chem. Phys. 2018, 20, 6800-6804.

(88) Würfel, P. Physics of Solar Cells: From Basic Principles to Advanced Concepts; Wiley-VCH Verlag $\mathrm{GmbH} \&$ Co.: Weinheim, 2005; p 71.

(89) Agiorgousis, M. L.; Sun, Y.-Y.; Zeng, H.; Zhang, S. Strong Covalency-Induced Recombination Centers in Perovskite Solar Cell Material CH3NH3PbI3. J. Am. Chem. Soc. 2014, 136, 14570-14575.

(90) Yin, W.-J.; Shi, T.; Yan, Y. Unusual defect physics in CH3NH3PbI3 perovskite solar cell absorber. Appl. Phys. Lett. 2014, 104, 063903.

(91) Du, M.-H. Density Functional Calculations of Native Defects in CH3NH3PbI3: Effects of Spin-Orbit Coupling and Self-Interaction Error. J. Phys. Chem. Lett. 2015, 6, 1461-1466. 